participants' language use is different from the dominant language of the country.

\section{OP86 UNDERSTANDING INTERVENTION ACCEPTABILITY: CHANGING PARTICIPANT VIEWS OVER TIME AND THE IMPORTANCE OF CONTEXT}

\begin{abstract}
${ }^{1} S$ Morgan-Trimmer*, ${ }^{2} \mathrm{~J}$ Gregory, ${ }^{3} \mathrm{~J}$ Townson, ${ }^{3} \mathrm{~S}$ Channon, ${ }^{4} \mathrm{C}$ Chestnutt, ${ }^{4} \mathrm{~B}$ Chadwick, ${ }^{5} \mathrm{~S}$ Murphy, ${ }^{6} \mathrm{~S}$ Hutchings. ${ }^{1}$ Psychology Applied to Health Group, University of Exeter Medical School, Exeter, UK; ${ }^{2}$ School of Medicine, Cardiff University, Cardiff, UK; ${ }^{3}$ Centre for Trials Research, Cardiff University, Cardiff, UK; ${ }^{4}$ School of Dentistry, Cardiff University, Cardiff, UK; ${ }^{5}$ Centre for the Development and Evaluation of Complex Interventions for Public Health Improvement (DECIPHer), School of Social Sciences, Cardiff University; ${ }^{6}$ Simbec-Orion Group Ltd
\end{abstract}

\subsection{6/jech-2017-SSMAbstracts.85}

Background Acceptability is an important aspect of the quality of health interventions and also has implications for the feasibility of future implementation. Process evaluations of complex interventions often assess the acceptability of interventions through qualitative interviews with participants, addressing participants' experience of, satisfaction with and preferences for treatment/services received. Acceptability can be influenced by multiple aspects of an intervention and its context. This paper reflects on two process evaluations which produced complex findings on acceptability: the Delivering Early Care In Diabetes Evaluation (DECIDE) study, a trial of home vs. hospital care following a diagnosis of Type I diabetes (T1D) in children, and the Seal or Varnish (SoV) study which compared fluoride varnish and fissure sealant dental treatments to prevent caries in children.

Methods In the DECIDE study, semi-structured interviews with 11 (pairs of) parents and seven children were conducted 15-20 months post-diagnosis about their experience of hospital or home care immediately following diagnosis of T1D. In the SoV study, all children completed a 'smiley face' acceptability questionnaire immediately after treatment. In addition, paired semi-structured interviews were conducted with children at the beginning (50 children) and end ( 32 children) of the intervention, within a few days of receiving treatment. Interviews were divided evenly between trial arms; questions included experience of and acceptability of the interventions, diabetes management (DECIDE) and management of dental health (SoV).

Results In the DECIDE study, most interviewees wanted to be randomised to the 'home' arm initially but expressed a retrospective preference for whichever trial arm they had been in. This shift in preference may have been influenced by 'positive attitude' coping strategies adopted by families. In the SoV study, acceptability immediately post-treatment was related to which treatment was received, but acceptability in the week following treatment was strongly influenced by wider aspects of treatment such as receiving a sticker and there was little difference in overall acceptability by trial arm. Perceptions of overall treatment may have been influenced by the intervention being delivered through a well-established, child-friendly dental service in a school setting.

Conclusion Both studies found that acceptability of an intervention can change over time, and indicated that participant restructuring of acceptability can be influenced by wider contextual factors of the intervention. Implications for future research are that the timing of data collection on acceptability may influence findings, and that acceptability (and implications for future implementation) should be interpreted in relation to intervention context.

\section{OP87 DAGITTY AND DIRECTED ACYCLIC GRAPHS IN OBSERVATIONAL RESEARCH: A CRITICAL REVIEW}

1,2PWG Tennant* ${ }^{3}{ }^{3}$ Textor, $1,4 \mathrm{MS}$ Gilthorpe, ${ }^{1,4} \mathrm{GTH}$ Ellison. 'Leeds Institute for Data Analytics, University of Leeds, Leeds, UK; ${ }^{2}$ School of Healthcare, University of Leeds, Leeds, UK; ${ }^{3}$ Department of Tumour Immunology, Radboud University Medical Centre, Nijmegen, The Netherlands; ${ }^{4}$ Leeds Institute for Cardiovascular and Metabolic Medicine, University of Leeds, Leeds, UK

\subsection{6/jech-2017-SSMAbstracts.86}

Background Empirical researchers working with observational data have been slow to adopt modern statistical methods for causal inference, which remain poorly recognised among applied quantitative researchers. First introduced in 2010, DAGitty is a free web application (and R package) that enables empirical researchers to draw directed acyclic graphs (DAGs) and identify minimally-sufficient adjustment sets without explicit knowledge of graphical model theory. This review examines empirical research articles that have used DAGitty as an aid for analysing observational data.

Methods Articles citing 'DAGitty' published before 1 July 2016 were identified through searching Web of Science, Medline, Scopus, PubMed, and Google Scholar. Original articles describing the analysis of observational data were identified by inspecting the published manuscripts. Information on the use and presentation of DAGs and adjustment sets were extracted into a standardised table. Bibliographic details (including journal discipline) were obtained from Thompson-Reuter's Journal Citations Reports.

Results 124 original articles describing the analysis of observational data were identified from 151 unique articles citing DAGitty. Two (2\%) were published in 2012, seven (6\%) in 2013, $23(19 \%)$ in 2014, $46(37 \%)$ in 2015, and $46(37 \%)$ in the first half of 2016. The first authors came from 18 countries, most commonly the USA $(n=36,29 \%)$, Germany $(n=19,15 \%)$, Australia $(n=14,11 \%)$, Sweden $(n=12,10 \%)$, the UK $(n=10,8 \%)$, and Denmark $(n=6,5 \%)$. The host journals represented 43 academic disciplines, most commonly 'Public, environmental, and occupational health' $(n=29,23 \%)$, 'environmental studies' ( $\mathrm{n}=13,10 \%)$, 'multidisciplinary sciences' $(\mathrm{n}=11,9 \%)$, 'oncology' $(\mathrm{n}=10,8 \%)$, 'nutrition and dietetics' $(\mathrm{n}=9,7 \%)$, and 'immunology' $(\mathrm{n}=8,6 \%)$.

$29(23 \%)$ articles included a DAG in the manuscript, 41 (33\%) in supplementary material, while 53 (44\%) contained no DAG. DAGs varied greatly in scope from three-variable overviews to graphs with $30+$ variables. Very few DAGs were saturated, whether completely or in order of transit. At the extreme, some researchers omitted all arcs except those that were explicitly evidenced. Adjustment sets were often modified beyond minimally-sufficient set(s) by adding: competing exposures (for 'improve precision'), mediators (to 'improve face val-

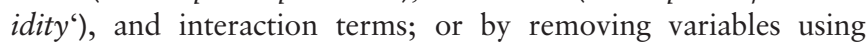
stepwise ( $\mathrm{p}$-value) methods or criteria for 'minimum change'.

Conclusion Use of DAGitty in empirical research is increasing exponentially. There is however huge variation in practice, with many choosing to blend DAG-based methods with more traditional/accepted approaches to model specification. Guidelines for 'best practice' should be developed and included in teaching material and/or journal guidelines. 